\title{
Enneking Stage IB
}

National Cancer Institute

\section{Source}

National Cancer Institute. Enneking Stage IB. NCI Thesaurus. Code C146702.

A stage for malignant musculoskeletal tumors characterized by low grade (G1), extracomparmental site (T2), and no metastasis (M0). 